\title{
Gobernabilidad en El Salvador: Ejercicio de medición de la administración de Francisco Flores*
}

\section{Introducción}

- Juan Carlos Morales Peña

El propósito de este trabajo es caracterizar la situación de gobernabilidad que se registra actualmente en El Salvador. Específicamente, se ha tomado como unidad de análisis temporal la administración de Francisco Flores. En el diseño de la estructura de este ensayo se explican en primer lugar los recursos del marco teórico utilizado desde la Ciencia Política y su contraste con las propuestas gubernamentales y los estudios de los círculos académicos salvadoreños en torno a este fenómeno. Este apartado incluye la problemática de la insuficiencia de estudios relacionados al tema de la gobernabilidad e identifica los principales ingredientes y componentes de análisis que por lo general sirven de herramientas en el diagnóstico que se genera en el ámbito político.

En segundo lugar se estableció el grado de gobernabilidad en el país de acuerdo a la propuesta académica del especialista Antonio Camou. Para ello se estudiaron algunas dinámicas de la comunidad política muy características del diseño institucional que se articuló en la fase de instauración. Acá se sostiene que El

* Trabajo presentado en el curso de Ciencia Política I del programa de Maestría en Ciencia Política de la Universidad Centroamericana "José Simeón Cañas", en mayo del presente año. 
Salvador posee un déficit de gobernabilidad que imposibilita que la sociedad dé un salto de calidad en materia sistémica, identificada ésta dentro de un contexto de cambio y permanencia.

En tercer lugar se profundizó en el examen de la evidencia, es decir, el diagnóstico de las zonas que generan luces rojas y déficits de gobernabilidad, unas con mayor incidencia que otras, pero, en definitiva, fundamentales para identificar los fenómenos que proporcionan respaldo al planteamiento del segundo apartado.

Un cuarto componente de este ensayo lo constituye el análisis del conflicto en los escenarios institucionales en donde se materializan los poderes del Estado, situación que no solamente reviste importancia por el hecho de que hace posible una aproximación al control del orden político, sino, además, permite identificar la lógica de las Administraciones de Gobierno en El Salvador y los conceptos de gobernabilidad desde la perspectiva de los enfoques oficiales y que impregnan el manejo de los asuntos nacionales. Para ello se tomaron cuatro casos al azar que tensaron en diversas coyunturas el debate legislativo. Dichos casos estuvieron referidos a la decisión de ratificar un Convenio que habilitaría la instalación de un centro de monitoreo en Comalapa administrado por autoridades militares norteamericanas para efectuar tareas de vigilancia de actividades ligadas a la narcoactividad; la ley de integración monetaria (popularmente conocida como dolarización), los intentos de regulación del margen de ganancias de las petroleras en el país y el veto presidencial en torno al ascenso a rango de General de un coronel del Ejército Salvadoreño.

Finalmente, se incluyen conclusiones que pretenden más que dar por agotado el examen de algunas problemáticas relacionadas a la gobernabilidad, advierten, por el contrario, los déficit de los que adolecen los estudios e investigaciones que en el país se hacen en torno a este fenómeno.

\section{Marco teórico versus realidad}

Antonio Camou, en su trabajo sobre gobernabilidad y democracia, identifica tres tradiciones o corrientes que explican las condiciones del fenómeno en cuestión'. Una de ellas de ellas incorpora la variable legitimidad como elemento fundamental para el buen gobierno; la segunda, utiliza la eficacia como variable para medir el grado de efectividad que se registra en el ejercicio del poder $y$, finalmente, una tercera, que se ubica en un nivel intermedio, pues incorpora elementos de las dos tradiciones anteriores pero a su vez identifica las propiedades de otras como la estabilidad; cuyos fundamentos son observados a partir de la capacidad de adaptación que un sistema es capaz de generar para responder a estímulos provenientes desde dentro y fuera de su entorno.

Por la naturaleza y los componentes identificados en el discurso oficial en torno a la gobernabilidad, la Administración Flores utiliza un enfoque cercano a esta última tradición en cuanto concibe en primer lugar la necesidad de estable- 
cer mecanismos estables de toma de decisiones; en segundo lugar, vincular el consenso de los grupos de poder económico (a los que se identifica como sociedad civil) al diseño institucional; y, en tercer lugar, utilizar recursos tales como pactos y arreglos para proporcionarle estabilidad a los objetivos de su gestión gubernamental. En síntesis, estas condiciones revelan el siguiente cuadro político: La Administración de Francisco Flores ubica la agenda nacional en el contexto de inserción del país en el fenómeno de globalización económica ${ }^{2}$, como fundamento de la política exterior que permite que el diseño institucional legado por la reforma política de postguerra genere una función de adaptación del sistema a las condiciones del exterior; esa agenda ha elaborado algunos elementos de contenido con los aportes de la sociedad civil desde la perspectiva gubernamental —entiéndase ésta última como la perspectiva de los grupos con poder económico que legitiman el programa de ajuste estructural- En otros términos, la lógica de la participación política definida desde arriba lo constituyen ejercicios limitados de consulta en temas claves de país que no se vinculan orgánicamente con el modelo económico ${ }^{3}$.

Algunos especialistas de los círculos académicos universitarios del país, como Óscar A. Fernández, hablan de niveles de relativización en la gobernabilidad de un sistema ${ }^{4}$, planteamiento que tiene como base la ubicación de los actores sociales; es decir, la conciencia de que determinados actores de la comunidad política tengan respecto a su influencia en el sistema. Si cada actor de la comunidad política tiene el control de determinadas variables del sistema, el fenómeno de la gobernabilidad se analizará en función de lo que la realidad permita a estos realizar en cuanto a sus objetivos específicos con el mínimo grado de conflicto. Un segundo nivel de relativización es el carácter de contenido del proyecto gubernamental, esto significa que en la medida este sea inclusivo o excluyente en esa medida capitalizará legitimidad hacia los objetivos de la gestión pública. Por ello, el programa político de las cuatro alianzas desarrolla su contenido en el contexto de una visión de país orientada a la inserción de éste a los procesos económicos mundiales y reconoce como actores primarios para este cambio de paradigma en lo que a desarrollo nacional respecta a los grupos sociales con poder económico 5 . El grado de vinculación de intereses entre éstos provee de estabilidad a la gestión gubernamental al menos en lo referente a las nuevas variables que sc incorporan al modelo económico a partir del diseño de política nacional que articula cada Administración. La actitud hacia el resto de actores de la comunidad política que no son concebidos como fundamentales para los objetivos de Gobierno la respuesta es diversa; una respuesta es contenerlos, es decir, mantenerlos al margen de las decisiones estratégicas de Gobierno que en teoría requerirían una base amplia de consenso; una segunda respuesta es incorporarlos en ejercicios limitados de consenso, en donde estén de por medio variables que no correspondan a la organización de la política económica. 
Un tercer nivel de relativización de la gobernabilidad del sistema gira en torno a la capacidad del ente gubernamental; si ésta se percibe como insuficiente, se genera una figura de gobierno débil y en esa medida puede incrementarse el nivel de demanda social y, sobre todo, si el nivel de respuesta no es el apropiado, lógicamente se gestarán conflictos.

En otros estudios sobre gobernabilidad —como el desarrollado por los especialistas Carlos Briones y Carlos G. Ramos en su libro La Gobermabilidad en Centroamérica: Gobernabilidad, Economía y Democracia en El Salvador - se plantea que la gobernabilidad se articula en las relaciones entre actores políticos, sociedad civil y gobierno dentro de un esquema dinámico en donde éste último hace que los dos primeros acepten las reglas y mecanismos institucionales establecidos que son los escenarios en donde se manifiestan todas las formas de ejercicio de poder en la sociedad; reconocen, asimismo, que una de las fuentes de la gobernabilidad se condiciona por la situación dinámica de las variables socioeconómicas.

En la idea básica de las características anteriores se incorpora también la condición de que las políticas públicas de una Administración de Gobierno puedan realizarse con niveles aceptables de legitimidad. En este componente es necesario identificar la dinámica de las variables sociales y económicas en el país y su impacto en la gobernabilidad. En retrospectiva, las Administraciones del partido ARENA, si bien han visto erosionada su legitimidad con el paso del tiempo, han mantenido el curso de los componentes de su programa de ajuste estructural, no obstante la oposición mostrada por otros actores de la comunidad política. En todo caso, la reforma económica ha condicionado su propio ritmo de celeridad en la medida las presiones de los diversos actores sociales se lo han permitido. Lo que sí cabe destacar es que la lógica del modelo económico no ha experimentado una ruptura orgánica y por lo tanto la tensión entre grupos sociales ha sido una constante que ha imposibilitado la construcción de una agenda que trascienda de una dimensión sectorial a una nacional.

Los estudios e investigaciones sobre gobernabilidad en el país aún no son suficientes y, además, adolecen de algunas limitaciones cuyo origen deriva también de los escasos estudios sobre el régimen político de postguerra, sino se tiene claro las características de este nuevo régimen no puede proporcionalmente entonces preverse una fórmula que permita definir y explicar la lógica de las demandas sociales y respuestas gubernamentales. Esto es fundamental para despojar a los programas de investigación existentes de la automaticidad de asociación de variables y condiciones como resultado de mecanismos que se programan en el espíritu del debe ser, sin consideración de otros elementos del sistema político que pesan en un proceso de cambio. Así, pues, asociar la democracia a la naturaleza del régimen y como tipificación que alude a la existencia de determinadas prácticas políticas, vincularla, además, a la necesidad de construcción del contenido de una nueva cultura política en menoscabo de los valores genera-

\section{0}

Realidad 81, 2001 
dos por la tradición y el proceso histórico y ligarla a la dinámica que caracteriza a la gobernabilidad ${ }^{7}$ produce, entre otros efectos:

a) En primer lugar, en el contexto de la investigación en ciencias sociales se produce un determinismo teórico, es decir, que, del resultado de asociar variables en la perspectiva de establecer puntos de llegada para un fenómeno político dentro de una lógica de reciprocidad, se le resta toda posibilidad de caracterización a la realidad y la ajusta a una dinámica insuficiente para explicar las situaciones problemáticas que se generan.

b) Es muy notable el hecho de que algunos especialistas incorporen en sus trabajos académicos las actitudes de los actores políticos en el período de transición, entendiéndose éste como la fase posterior a los Acuerdos de $\mathrm{Paz}^{8}$. Esta contextualización de las categorías de análisis impide resolver y ponderar en su justa dimensión las anomalías que se registran en las relaciones de poder entre los grupos sociales y las administraciones de gobierno.

c) Finalmente, en la medida que el déficit de gobernabilidad siga asociándose a una situación de desarrollo de la democracia, en esa proporción se marginará del análisis de las variables del sistema que intervienen para medir la gobernabilidad otros elementos que, por su peso, pueden explicar el comportamiento de los grupos sociales en la lógica del diseño institucional articulado para el Estado en la postguerra.

\section{El déficit de gobernabilidad en El Salvador}

Los niveles de respuesta gubernamental de la Administración Flores a las demandas sociales han sido insuficientes lo que ha permitido que dicha relación se encuentre en desequilibrio. La última encuesta del Instituto Universitario de Opinión Pública de la UCA (IUDOP) señala que el presidente hizo un buen manejo de la crisis durante el período de emergencia ocasionado por los terremotos de enero y febrero". No obstante ese dato, ha sido evidente la incapacidad gubernamental para resolver crisis coyunturales en un corto plazo, con el mínimo de consenso y libre de problemas colaterales que son las principales características que han definido las decisiones gubernamentales frente a las demandas de otros actores como el gremio de médicos del ISSS en torno a la crisis del sector salud y otros como el de APROAS de exdesmovilizados de la antigua Defensa Civil y patrullas cantonales, entre otros casos. La característica primera de estas crisis así como otras similares es que no han sido resueltas en un corto plazo, por lo general la Administración Flores ha ejercido una política de no negociar bajo presión, de establecer un canal de diálogo únicamente si las acciones del grupo de presión cesan previamente y la tendencia a descalificar a cualquier interlocutor que ofrezca interponer sus buenos oficios para la resolución de la crisis; además, ante la imposibilidad de disuadir al actor que impulsa una demanda, la 
Administración articula la divulgación de un diagnóstico y lectura del problema a través de los medios de comunicación en donde se trata de vincular la crisis a un chivo expiatorio que se utiliza en la dinámica de un fenómeno parapolítico (Es común culpar a la oposición en este tipo de coyuntura).

En cuanto a la segunda característica, la disolución de la crisis se ha hecho con un mínimo de consenso, dentro de un proceso tardío y únicamente cuando ésta amenaza con generar consecuencias para el partido oficial. El diagnóstico de la Administración Flores en torno a las demandas de diversos grupos de la sociedad presenta entre otros rasgos; el de calificarlos de ilegítimos si estos provienen de sectores allegados a determinados grupos de oposición, esto hace que las actitudes iniciales se generen en el marco de un diagnóstico que considera que detrás de toda demanda existe un nivel de manipulación determinado. La lógica de explicar las crisis utilizando chivos expiatorios busca crear la percepción de que la gestión publica esta bien, pero ésta se convierte en blanco de los grupos opositores. Además, el nivel de respuesta no es el apropiado de acuerdo al nivel de crisis que se registra y es desigual en cuanto concede en mayor medida a las presiones que provienen de actores con poder económico (Gobierno de EE.UU., petroleras, cafetaleros, industriales avícolas, etc.)

La crisis bajo estas condiciones han sido disueltas con instrumentos que incluyen arreglos políticos (caso de la huelga de médicos del ISSS) ${ }^{10}$, o bien, la eficiencia del recurso fuerza como es el caso de los ex patrulleros. Es notable que estas situaciones problemáticas en su contenido han sido reconvertidas a otros escenarios y mecanismos para su seguimiento y por ello no se puede hablar de resolución de una crisis.

La tercera característica es que algunas de las crisis registradas en el período de la Administración Flores han generado por lo general problemas colaterales que incluyen, entre otros, el que la crisis produzca una reacción en cadena en otros sectores de la sociedad que advierten en la dinámica de presión y respuesta al problema, el escenario de oportunidad para organizar su agenda sectorial en una demanda social concreta; las repercusiones de crisis largas han tenido implicaciones políticas en la correlación de fuerzas entre los sectores oficiales y la oposición y por lo tanto es suficiente para revelar la naturaleza de los ciclos de enfrentamiento.

Según la propuesta académica de Antonio Camou referente a los grados de gobernabilidad en una sociedad, cuando la percepción de algunos actores políticos organizados reprueba el desequilibrio existente entre el nivel de demandas sociales y respuestas gubernamentales. Estos hacen uso de sus capacidades para amenazar la relación de Gobierno. Este es el escenario más aplicable para la construcción de un ejercicio hacia la realidad nacional; No obstante, Camou propone otros niveles, como el de gobernabilidad ideal e ingobernabilidad, que son conceptos límite para hacer factible la designación de dimensiones con características intermedias. El déficit de gobernabilidad es el caso de El Salvador porque las anomalías 
que sc registran en la relación demanda social-respuesta gubernamental, no son resucltas en una dimensión proporcional. Es común que se manifiesten respuestas gubernamentales deficientes que desactivan la crisis coyuntural y sea reconvertida, o. en todo caso, marginada de la agenda de gobierno; desde el ámbito de la demanda social también existe la conformación de una agenda básica que se materializa de acucrdo al nivel de concesión que los grupos de presión obtienen de cada crisis coyuntural. La crisis es, así, un recurso de objetivos limitados, en donde no se comprometen las prioridades definidas por los grupos sociales en su agenda sectorial.

El délicit de gobernabilidad en El Salvador durante la Administración Flores se asocia a la lógica de tres fenómenos presentes en la mayoría de las crisis registradas. En primer lugar, la tendencia, por parte del ente gubernamental, de despolitizar la crisis mediante la deslegitimación de la demanda social. En la medida en que la lógica de los escenarios institucionales en donde se ventila la crisis y las actitudes de los actores involucrados se circunscriban a una dimensión técnico administrativa, en esa medida el Gobierno contiene el desgaste y los costos políticos, si es imposible recrear el tratamiento a la crisis en esas condiciones, se recurre entonces a la movilización de los enfoques bipolares que articulan el antagonismo histórico entre el partido ARENA y el FMLN como una forma de proporcionar identidad a sus opciones y desacreditar las del adversario.

Una segunda tendencia que incorpora la lógica de la idea anterior es la actitud del partido FMLN de utilizar la crisis coyuntural para bloquear o presionar a los objetivos de la gestión gubernamental y crear costos políticos para el partido oficial. La crisis en sí implica que es un escenario para medir fuerzas, propuestas e ideas; y, más importante aún, es tomar el pulso de la opinión pública en torno a determinados asuntos. En todo caso, la dinámica de enfrentamiento bipolar hace posible que los dos actores principales del espectro político marginen las posibilidades de terceros y capitalicen para sus propuestas los resultados que la crisis estimula en la opinión pública y anticipar así determinados cursos de acción.

El tercer fenómeno condicionante es el referido a la pasividad de la sociedad civil, entendida ésta en un sentido lato. Esto tiene un vínculo orgánico importante con el primer fenómeno: a mayor despolitización, menor ejercicio de fiscalización de la gestión pública por parte de la ciudadanía; y, por el contrario, a mayor politización, se incrementa la respuesta ciudadana para hacer o participar en política."

De tal forma, en ciertas crisis, algunos actores de la comunidad política han ejercido una notable capacidad para amenazar las relaciones de gobierno con las diferentes esferas de la sociedad, y sus formas de manifestación han incluido el bloqueo, dilatación, contrapropuesta, protesta, movilización, entre otros.

Nótese que las presiones hacia el programa de ajuste estructural en su mayoría han dilatado la implementación de medidas, pero no las han revocado o revertido; en todo caso, la zona de la economía es en donde se gestan buena parte de las tensiones entre los actores de la comunidad política. 


\section{Zonas sistémicas: La intensidad de las luces rojas}

Siguiendo la propuesta de Antonio Camou, el déficit de gobernabilidad puede ubicarse en diversas zonas del sistema. Cada una articula su propio peso y dimensión en su vínculo con los objetivos de una Administración de Gobierno y cl nivel de respuesta que proporciona hacia las demandas del resto de la comunidad política.

En cuanto al orden público (como primera zona sistémica), si un gobierno es sobrcpasado por la actividad de otros grupos que, independientemente de la lógica que articulen, crean ruptura en las condiciones de garantía para el normal desarrollo de las actividades cotidianas de la sociedad, se crea una percepción en el público en donde se ubica al ente gubernamental en una situación de debilidad firente a otros actores.

Durante la Administración Flores, la ciudadanía sigue estando preocupada por la violencia de la delincuencia común y el crimen organizado, se percibe como una amenaza para la autoridad del Estado después de la crisis económica $^{12}$. El crimen organizado cuenta con la capacidad para infiltrar las instituciones del Estado, alterar la ética que puede privar en la gestión pública y estos aspectos minan el nivel de confianza de los ciudadanos en las instituciones responsables del orden público (PNC, Fiscalía, Sector Justicia, etc.)

Una segunda zona sistémica, la economía, es actualmente la mayor preocupación de los salvadoreños. En la percepción colectiva, el éxito macroeconómico no se corresponde a la precaria situación de la economía familiar. Las variables pobreza, desempleo y falta de oportunidades constituyen el principal estímulo del desánimo social ${ }^{13}$. Y, por ello, algunas respuestas sociales son concomitantes a los escasos espacios de materialización de los objetivos de la Administración Flores en materia de política económica. En lo que respecta al modelo económico ha sido común en las Administraciones del partido ARENA, el no propiciar consultas ciudadanas ni mucho menos capitalizar determinada legitimidad hacia las medidas de refuerzo del programa de ajuste estructural, a excepción de los grupos de poder económico que tienen influencia sobre el Gobierno.

La tercera zona sistémica, muy ligada a la segunda, es la del bienestar social. La reforma económica en el país está articulada en la estructura de su diseño por cl programa de ajuste estructural y los objetivos de éste son, entre otras cosas, desmantelar el capitalismo de Estado, convirtiendo este último en un ente normativo del libre juego de las fuerzas del mercado. Esto significa que algunos recursos de política económica utilizados tales como del libre juego de las fuerzas del mercado. Esto significa que algunos recursos de política económica utilizados, tales como la privatización de las empresas de servicios públicos y la desgravación arancelaria están orientados a descargar de las instituciones una serie de responsabilidades sociales que en el actual contexto se transfieren al sector privado con la orientación de hacer factible la modernización de estos servicios. La lógica es 
acelerar la crisis del Estado de bienestar ${ }^{14}$ social: el servicio tiene un costo y el ciudadano debe pagarlo en aras de incrementar su calidad. Esto es imposible si este se provee desde el Estado, pero sí tiene mayores márgenes de mejora, si es suministrado por la empresa privada. Éstas son algunas razones de justilicación de las medidas del programa de ajuste estructural.

La cuarta zona sistémica se ha analizado a partir de un ejercicio de medición de la Administración Flores con respecto a algunas crisis registradas en su período, el ámbito se ubica en el grado de control del orden político que éste ha ejercido durante casi dos años de gestión. Este análisis constituye el siguiente apartado de este ensayo.

\section{El control del orden político: El conflicto en los escenarios institucionales}

Después del último evento electoral (marzo de 2000), en el que se eligieron nuevas autoridades legislativas y consejos municipales, el partido ARENA, pese a registrar la mayor cantidad de votos válidos durante el escrutinio, no logró consolidar una representación mayoritaria en la Asamblea Legislativa, lo cual le hubiese posibilitado una agilización de la agenda del Ejecutivo. Esta situación ha sido resuelta en la práctica política con el acercamiento a otros partidos de derecha con los que existe afinidad en las plataformas programáticas, en este caso, el PCN. Tambien se han dado acercamịentos con el PDC, en algunas oportunidades.

La aritmética legislativa ha favorecido a los objetivos de gestión de la Administración Flores sobre todo en materia económica. La Asamblea Legislativa se ha convertido en uno de los escenarios institucionales en donde más se han generado tensiones en relación con las decisiones e iniciativas provenientes del Ejecutivo.

En marzo del año 2000, el Ejecutivo, a través del Ministerio de Relaciones Exteriores, firma un convenio de cooperación con el Gobierno de los Estados Unidos, que permite a este último instalar un centro de monitoreo para el desarrollo de actividades de vigilancia y reconocimiento de vuelos y embarcaciones ilegales, sospechosas de transportar cargamentos con drogas. El debate posterior giró en torno a las consecuencias sobre la soberanía nacional que dicho convenio ocasionaría si fuese ratificado ${ }^{15}$. Los elementos que son importantes observar en todo caso es el hecho de cómo a partir de una aritmética legislativa favorable, el Gobierno logra movilizar sus objetivos en esta iniciativa y por lo tanto capitalizar artificialmente algún grado de legitimidad a su decisión en esta materia. El caso del convenio de cooperación antinarcotráfico no degeneró en crisis, si bien registró significativos niveles de tensión. El éxito gubernamental se debió en primer lugar en haber utilizado los recursos legales previstos por las instituciones para revestir la decisión tomada, el conflicto de esta iniciativa con la ley primaria se resolvió con anticipación, si bien las interpretaciones constitucionales expuestas no aclararon cuestiones fundamentales inmersas en el proceso de ratificación. El

Gobernabilidad en El Salvador 
Gobierno logra convencer a varias fracciones legislativas (PCN, PDC y PAN) y esto materializa una cantidad de votos suficientes para aislar a la izquierda (FMLN). No fue una crisis dentro del control del orden político, porque la tensión sc limitó al escenario legislativo. Los opositores al convenio no tuvieron la capacidad de movilizar a otros sectores para protestar por la ratificación; una vez el convenio entró en vigencia, los recursos institucionales para bloquearlo, e incluso, revertirlo, se redujeron al ámbito cle los escenarios judiciales.

En el caso de la dolarización privó también la aritmética legislativa favorable al Gobierno, pero, por el carácter sorpresivo e inconsulto de la iniciativa, esta enfrentó mayores grados de rechazo y oposición. La libre circulación del dólar necesitaría algo más que su respaldo legal por parte de las instituciones involucradas del Estado. Tras la ratificación de la Ley de Integración Monetaria, la oposición logró crear un consenso básico en algunos núcleos de la población para el rechazo de la medida. La crisis se genera cuando sectores importantes de la sociedad rechazan la medida y amenazan con bloquearla. En muchos consejos municipales presididos por el FMLN se planteó no recibir dólares y los transportistas también adoptan una actitud de bloqueo aduciendo pérdidas económicas importantes para su gremio, etc. En general, la dolarización de la economía estimula una crisis que es desactivada por la coyuntura de la emergencia nacional siguiendo la lógica identificada en la Administración Flores, las medidas e instrumentos de política económica no se negocian con la oposición. No obstante lo anterior, buena parte de la población incrementa su desconfianza a la medi$\mathrm{da}$, si se tiene en cuenta que algunos beneficios sostenidos en el discurso oficial no parecen estarse generando ${ }^{16}$.

Algunas iniciativas del Ejecutivo tuvieron también ciertos problemas de correspondencia con la fracción legislativa del partido of icial, como la iniciativa (decreto) para reducir el margen de ganancia de las petroleras en. el país. Esta crisis definió algunos elementos novedosos en lo que a su proceso respecta. En primer lugar, ante la enornne presión de las transnacionales (Esso, Texaco y Shell), el Gobierno de Flores consolida su peso ante la demanda, incorporando el apoyo de las fracciones legislativas que mostraron su acuerdo con el decreto. En este contexto, se producen las diferencias con la fracción de ARENA que nunca se mostró convencida para apoyar la iniciativa presidencial. Ello estimuló a las petroleras a obligar un espacio para la negociación, condición que permitió que el espíritu del decreto para favorecer al consumidor de combustible perdiera consistencia. Las petroleras logran excluir del diagnóstico oficial referente a las alzas de los precios de los combustibles, a los márgenes de ganancias, propician un debate que hace que el diagnóstico de ese problema incorpore y vuelva más fundamentales otras variables tales como la inestabilidad del mercado de energéticos, el subsidio al transporte colectivo, entre otros. Cierra el círculo de la negociación, lanzando una iniciativa unilateral de buena voluntad consistente en reducir los precios de los combustibles por un período específico y orientar así 
el debate en torno al problema hacia otros componentes parte del diagnóstico; como una actitud dirigida a legitimar su enfoque.

Un cuarto caso en un escenario diferente lo constituyó el intento de algunos partidos políticos de oposición de cuestionar los criterios presidenciales que denegaron el ascenso a General del coronel Munguía Payés por segunda ocasión. La supuesta violación de los procedimientos del Tribunal de Ascensos y los esfuerzos para superar el veto presidencial en torno a este caso revelan dos ingredientes: en el primero, se cuestiona la primacía del poder civil sobre el militar, en un contexto de régimen en donde la lógica de funcionamiento prevista para estas decisiones define un carácter de subordinación. El problema estriba en el grado de subjetividad, desconocimiento y apreciación política que pueden influir en la decisión de un presidente para negar o permitir el ascenso de los oficiales de las Fuerzas Armadas, de allí deriva la tensión en el caso expuesto.

Por su parte, el otro ingrediente se ubica en el contexto de medición de fuerzas entre Gobierno y oposición legislativa en torno al problema de los ascensos, en el cual se coloca al estamento militar en un dilema: El de la apoliticidad y no deliberación, principios que son mantenidos mediante la decisión de la cúpula castrense de sujetarse a la decisión presidencial. La situación problemática sigue latente, sin embargo, por el hecho de que el estamento militar pudo advertir una severa presión política sobre sus intereses corporativos, y ante el manejo que los partidos políticos hicieron de este escenario, los militares habrán identificado los riesgos de participar como instrumento de definición en las tensiones que producen las correlaciones de fuerza entre partidos.

El control del orden político presenta déficit importantes en el sentido de que un Gobierno no puede realizar sus objetivos de gestión, si no posee mayoría oficial en la Asamblea o articule por otra parte un consenso básico que le permita movilizar sus iniciativas. Cabe destacar que la aritmética legislativa no ha favorecido siempre a la Administración Flores, pues la oposición ha ejercido una significativa capacidad de bloqueo en otras áreas como las relacionadas a la aprobación y ratificación de préstamos internacionales.

\section{Conclusiones}

(1) La Administración de Francisco Flores se mueve en torno a ejes de discusión que tienen que ver con la inserción del país al proceso de globalización económica. En este sentido, la captación de beneficios que en teoría provendrían de dicha decisión definiría los factores de estabilidad del modelo político. El principio rector se orienta a que el proceso de inserción sea un fenómeno irreversible y sea por lo tanto asumido por futuros gobiernos. En el fondo, se trata de proporcionar estabilidad a las instituciones del Estado a partir de una fórmula que despeje la ecuación político partidaria actual a favor de acuerdos básicos que garanticen la continuidad del sistema, principalmente en su zona económi- 
ca; este sería el escenario en un contexto de relevo en el gobierno por parte de un partido político de oposición.

(2) La variable democracia sigue utilizándose en el marco de una asociación automática en la mayoría de los programas de investigación én los círculos académicos del país. De esta manera, los estudios sobre gobernabilidad no aportan fómmulas de funcionamiento para materializar un equilibrio entre demandas sociales y respuesta gubernamental. Algunos de los trabajos analizados en esta línea ajustan los valores de la democracia como fenómeno ideal y en el ejercido de forzar determinadas prácticas, se margina la lógica en las que se dinamizan los conflictos en la sociedad. Al igual que las contradicciones que genera la implantación de un modelo económico que desconoce los déficits estructurales y obliga a los procesos internos hacia transformaciones que no producen los resullados esperados; de igual forma, la crisis en las diversas zonas sistémicas debe resolverse con instrumentos funcionales en donde se incorporen las características de vinculación propias a la cultura y formas tradicionales entre grupos sociales, partidos y gobierno y estas no siempre son el producto de ideales.

(3) La Administración Flores posee una relativa estabilidad para realizar sus objelivos de gestión. Esa relatividad viene dada por una variable dependiente que hace que el Ejecutivo requiera del acompañamiento de un socio menor al interior de la Asamblea Legislativa. Esto, para concretar aritméticas legislativas que le sean favorables para agilizar su agenda. Por depender de la actitud de un socio menor - que en este contexto se ha identificado en el PCN - la capacidad de chantaje de éste se orientará más allá de lograr específicas cuolas de poder y reproducir las condiciones del modelo político que le han garantizado su permanencia en el espectro político durante mucho tiempo.

(4) Tanto la oposición encabezada por el FMLN como el sector oficial no poseen la suficiente capacidad para bloquear o generar la parálisis institucional que impida el curso de sus iniciativas. Acá se incorpora el elemento tripolar, puesto que ambas fuerzas mayoritarias dependen de los arreglos con fuerzas políticas menores al interior cle la Asamblea para materializar una correlación de fuerzas que les permita sobre ponerse a la opción del adversario. Esos arreglos han favorecido al Gobierno en la mayoría de los casos a excepción de lo referente a ratificación y aprobación de préstamos internacionales, rubro estratégico si sc entiende que su bloqueo implica privar al Ejecutivo de importantes recursos cconómicos.

\section{Notas}

1. Antonio Camou caracteriza con mayor profundidad estas tradiciones en un cuaderno de trabajo de divulgación de la cultura democrática, auspiciado por el Instituto Federal Electoral de México. La eficacia, la legitimidad y la estabilidad son las variables 
principales de medición para registrar los grados de gobernabilidad en una sociedad.

2. Flores plantea ese paradigma como fundamento de las posibilidades de desarrollo del país, y expone su justificación en un discurso pronunciado en Nueva York el 6 de septiembre de 2000. En él reconoce que la apertura económica, el acceso a mercados y la eliminación de todas las formas de asistencialismo son claves para la inserción del aparato productivo nacional al fenómeno de la globalización económica.

3. Ese es el caso de las mesas de trabajo que se organizaron para proveer de insumos al documento del Plan de Nación, en donde surgieron puntos referidos al ordenamiento territorial, la cuestión educativa, la zonificación del país, etc. Sin embargo, no se advierten componentes referidos al modelo económico.

4. Óscar A. Fernández expone estos niveles y establece un vínculo de los mismos con el rubro de Seguridad Pública en un ensayo titulado Seguridad pública y gobernabilidad democrática, Revista Entorno de la Universidad Tecnológica de El Salvador (UTEC), octubre de 1998, Núm. VII, Pág. 74

5. Flores hace esa identificación durante la exposición del discurso de su inauguración en el gobierno; allí reconoce el papel de los agentes económicos financieros, empresariales, gremiales y otros a los que hizo un llamado a incorporarse al programa de las cuatro alianzas para generar un futuro de creación de riquezas, empleos y oportunidades.

6. Este trabajo fue auspiciado por FLACSO y en él se analiza el fenómeno de la gobernabilidad, tomando como períodos las dos primeras Administraciones del partido ARENA.

7. Carlos Briones hace dicha asociación en un planteamiento al que dedica un capítulo entero en su obra. Por ello, ese criterio de asociación entre las variables gobernabilidad y democracia ha influido en buena parte los programas de investigación dedicados al estudio del fenómeno de la gobernabilidad.

8. Carlos Briones, en la parte V de Las actitudes políticas en la transición: ¿Rechazo del pasado o transformación de la cultura política?, identifica dicho período en la fase posterior a los acuerdos de paz. Otros especialistas desde la perspectiva de la Ciencia Política sitúan su inicio en la ruptura del régimen autoritario presidido por los militares (específicamente el golpe de Estado de octubre de 1979) y finalizan con la firma de los acuerdos de paz en enero de 1992.

9. Aproximadamente un $61.2 \%$ de las mil 222 personas consultadas aprueba en términos positivos la conducción de Flores de la situación de emergencia. Los resultados de la encuesta del IUDOP aparecieron en la sección de Nacionales del rotativo La Prensa Gráfica, el jueves 10 de mayo, p. 40.

1). La huelga de médicos y trabajadores del ISSS que duró cuatro meses y medio fue resuelta mediante un arreglo político que involucró, en la negociación secreta que se realizo, al Gobierno, FMLN y representantes del sector en conflicto. Los acuerdos prevén seguir ventilando las diferencias en los escenarios institucionales correspondientes y a otro nivel.

11. El analista Dagoberto Gutiérrez desarrolla estas ideas basándose en las experiencias a nivel político que se han observado en numerosos procesos de transición alrededor del mundo. Esta exposición se desarrolló en el marco de un panel foro denominado Análisis de la Coyınntura Política Acıual, organizado en marzo del presente año por el Colegio de Altos Estudios Estratégicos (CAEE) como parte del plan de esiudios del X Curso de Seguridad Nacional y Desarrollo.

12. Encuesta del IUDOP, cilada en La Prensa Grófica, Ibid., p. 5 


\section{Idem.}

14. Camou reconoce esta tendencia en su introducción del documento Gobernabilidad y democracia, cuando identifica el agotamiento estructural del modelo del Estado interventor y su consiguiente reforma.

15. Esa es una de las principales conclusiones emanadas de las mesas de trabajo en las que participó el equipo multidisciplinario de asesores académicos del Colegio de Altos Estudios Estratégicos, en julio de 2000. El resultado de las sesiones de discusión fue el documento de diagnóstico parcial intitulado El dispositivo antinarcotráfico de los Estados Unidos en América Central y sus implicaciones en la seguridad nacional de El Salvador.

16. La afirmación retoma las posturas y análisis de muchos especialistas y representantes de sectores, entrevistados en los medios de comunicación social para analizar el impacto de la dolarización en sus sectores y actividades productivas, durante el período de enero de 2001 . 